EPJ Web of Conferences 73, 03017 (2014)

DOI: $10.1051 /$ epjconf/20147303017

(C) Owned by the authors, published by EDP Sciences, 2014

\title{
Spin-1 particles with light-front approach
}

\author{
J.P.B.C. de Melo ${ }^{1, a}$, Anacé N. da Silva ${ }^{1}$, Clayton S. Mello ${ }^{1,2}$ and Tobias Frederico ${ }^{2}$ \\ ${ }^{1}$ Laboratório de Física Teórica e Computacional, LFTC, Universidade Cruzeiro do Sul, \\ São Paulo 01506-000, Brazil \\ ${ }^{2}$ Departamento de Física, Instituto Tecnológico de Aeronáutica, Centro Técnico Aeroespacial, \\ 12.228-900, São José dos Campos, São Paulo, Brazil
}

\begin{abstract}
For the vector sector, i.e, mesons with spin-1, the electromagnetic form factors and anothers observables are calculated with the light-front approach. However, the lightfront quantum field theory have some problems, for example, the rotational symmetry breaking. We solve that problem added the zero modes contribuition to the matrix elements of the electromagnetic current, besides the valence contribuition. We found that among the four independent matrix elements of the plus component in the light-front helicity basis only the $0 \rightarrow 0$ one carries zero mode contributions.
\end{abstract}

\section{Introduction}

The main objective of the $Q C D$, (quantum cromodynamics) quantum field theory is explain the bound state hadronic structure; mesons and baryons, in terms of the fundamental block of the matter, i.e., quarks and gluons. However, it is no simple task in the quantum field theory [1]. By another side, the strong interactions of the hadrons, is not described by the perturbative quantum field theory. The alternative is the light-front quantum field theory ( $L F Q F T)$, the natural approach to hadronic bound state systems [2], the first time, formulated by Paul Dirac some years ago [3]. The spin-1 particles have an more complicate structure with compared hadronic spin zero structure, for example the pion meson and kaon [4-10]. However in the last years, many works dedicate to the structure of spin-1 particles appears with differents approachs (see the references [11-18, 20, 21]). In this work, we use an light-front constituent quark model (LFCQM) [11] in order to calculate some observables for spin-1 particle, i.e, the rho meson. However, with the light-front approach, besides the valence components of the electromagnetic current, in order to keep the covariance, is necessary to add the non-valence components of the electromagnetic current $[5,11]$.

\footnotetext{
ae-mail: joao.mello@cruzeirodosul.edu.br
}

This is an Open Access article distributed under the terms of the Creative Commons Attribution License 4.0, which permits unrestricted use, distribution, and reproduction in any medium, provided the original work is properly cited. 


\section{The model}

The matrix elements of the electromagnetic current $\mathcal{J}_{j i}=\epsilon^{\prime}{ }^{\alpha} \epsilon_{i}^{\beta} J_{\alpha \beta}^{\mu}$ for spin-1 particles, in the impulse approximation are written as [11], for the plus component of the matrix elements of the electromagnetic current:

$$
\begin{aligned}
J_{j i}^{+}= & \iota \int \frac{d^{4} k}{(2 \pi)^{4}} \frac{\operatorname{Tr}\left[\epsilon_{j}^{\prime \nu} \Gamma_{\beta}\left(k, k-p_{f}\right)\left(k-p_{f}+m\right) \gamma^{+}\right.}{\left(\left(k-p_{i}\right)^{2}-m^{2}+\imath \epsilon\right)\left(k^{2}-m^{2}+\imath \epsilon\right)} \\
& \frac{\left.\left(k-l p_{i}+m\right) \epsilon_{i}^{\mu} \Gamma_{\alpha}\left(k, k-p_{i}\right)(k+m)\right]}{\left(\left(k-p_{f}\right)^{2}-m^{2}+\imath \epsilon\right)} \\
& \times \Lambda\left(k, p_{f}\right) \Lambda\left(k, p_{i}\right),
\end{aligned}
$$

where $\epsilon_{j}^{\prime v}$ and $\epsilon_{i}^{\mu}$ are the polarization four-vectors of the final and initial states, respectively and $m$ is the constituent quark mass. In the equation above, the $\Lambda(k, p)=1 /\left((k-p)^{2}-m_{R}^{2}+\imath \epsilon\right)$, is the regulator function in order to turn the Feynman amplitude finite. The explicity polarizations used here, are $\epsilon_{x}^{\mu}=(-\sqrt{\eta}, \sqrt{1+\eta}, 0,0), \epsilon_{y}^{\mu}=(0,0,1,0), \epsilon_{z}^{\mu}=(0,0,0,1)$, for the initial state and the final state, $\epsilon_{x}^{\prime \mu}=(\sqrt{\eta}, \sqrt{1+\eta}, 0,0), \epsilon_{y}^{\prime \mu}=(0,0,1,0), \epsilon_{z}^{\prime \mu}=(0,0,0,1)$. The vertex model to the bound state spin-1 particle and pair $q \bar{q}$ utilized here is [11]:

$$
\Gamma^{\mu}(k, p)=\gamma^{\mu}-\frac{m_{v}}{2} \frac{2 k^{\mu}-p^{\mu}}{p \cdot k+m_{v} m-\imath \epsilon},
$$

the vector particle is on-shell and $m_{v}$ is the vector bound state mass. After the integration in the lightfront energy, $k^{-}=\frac{\vec{k}_{\perp}^{2}+m^{2}}{k^{+}}$, the light-front valence wave function is obtained:

$$
\Phi_{i}\left(x, \vec{k}_{\perp}\right)=\frac{N^{2}}{(1-x)^{2}\left(m_{v}^{2}-M_{0}^{2}\right)\left(m_{v}^{2}-M_{R}^{2}\right)^{2}} \vec{\epsilon}_{i} \cdot\left[\vec{\gamma}-\frac{\vec{k}}{\frac{M_{0}}{2}+m}\right] .
$$

\section{Electromagnetic form factors}

The electromagnetic form factors for spin-1 particles, are linear combinations of the electromagnetic current $[11,22]$. However, for spin-1 particles, we have four matrix elements and only three electromagnetic form factors, $G_{0}, G_{1}$ and $G_{2}$, ie., charge, magnetic and quadrupole electromagnetic form factors respectively, then, the combinations of the matrix elements to extract the electromagnetic form factors, present some arbitrary in the LFQFT.

But, in the LFQFT, thanks by the the angular condition [22, 23],

$$
\begin{aligned}
\Delta\left(q^{2}\right) & =(1+2 \eta) I_{11}^{+}+I_{1-1}^{+}-\sqrt{8 \eta} I_{10}^{+}-I_{00}^{+} \\
& =\left(J_{y y}^{+}-J_{z z}^{+}\right)(1+\eta)
\end{aligned}
$$

it is possible eliminate some matrix elements of the electromagnetic current and extract the electromagnetic form factors. In the literature, we have four prescriptions to combine the matrix elements of the electromagnetic current in order to calculate the electromagnetic form factors [11, 22], using the valence part of the electromagnetic current.

In this work, we use two availables prescriptions in the literature $[23,24]$ and compare that prescriptions with the instant form basis. In the case, of the reference [23], the elimination of matrix 
elements $I_{00}^{+}$, produce the following expression to the electromagnetic form factors:

$$
\begin{aligned}
G_{0}^{G K} & =\frac{1}{3}\left[(3-2 \eta) I_{11}^{+}+2 \sqrt{2 \eta} I_{10}^{+}+I_{1-1}^{+}\right] \\
& =\frac{1}{3}\left[J_{x x}^{+}+2 J_{y y}^{+}-\eta J_{y y}^{+}+\eta J_{z z}^{+}\right], \\
G_{1}^{G K} & =2\left[I_{11}^{+}-\frac{1}{\sqrt{2 \eta}} I_{10}^{+}\right] \\
& =J_{y y}^{+}-\left(J_{z z}^{+}+\frac{J_{z x}^{+}}{\sqrt{\eta}}\right), \\
G_{2}^{G K} & =\frac{2 \sqrt{2}}{3}\left[-\eta I_{11}^{+}+\sqrt{2 \eta} I_{10}^{+}-I_{1-1}^{+}\right] \\
& =\frac{\sqrt{2}}{3}\left[J_{x x}^{+}+J_{y y}^{+}(-1-\eta)+\eta J_{z z}^{+}\right] .
\end{aligned}
$$

In the reference [24], the $I_{11}^{+}$matrix element of the electromagnetic current is eliminate from Eq. (4), and the eletromagnetic form factors to spin-1 particles are given by:

$$
\begin{aligned}
G_{0}^{B H} & =\frac{1}{3(1+\eta)}\left[(3-2 \eta) I_{00}^{+}+8 \sqrt{2 \eta} I_{10}^{+}+2(2 \eta-1) I_{1-1}^{+}\right] \\
& =\frac{1}{3(1+2 \eta)}\left[J_{x x}^{+}(1+2 \eta)+J_{y y}^{+}(2 \eta-1)+J_{z z}^{+}(3+2 \eta)\right] \\
G_{1}^{B H} & =\frac{2}{(1+2 \eta)}\left[I_{00}^{+}-I_{1-1}^{+}+\frac{(2 \eta-1)}{\sqrt{2 \eta}} I_{10}^{+}\right] \\
& =\frac{1}{(1+2 \eta)}\left[\frac{J_{z x}^{+}}{\sqrt{\eta}}(1+2 \eta)-J_{y y}^{+}+J_{z z}^{+}\right] \\
G_{2}^{B H} & =\frac{2 \sqrt{2}}{3(1+2 \eta)}\left[\sqrt{2 \eta} I_{10}^{+}-\eta I_{00}^{+}-(\eta+1) I_{1-1}^{+}\right] \\
& =\frac{\sqrt{2}}{3(1+2 \eta)}\left[J_{x x}^{+}(1+2 \eta)-J_{y y}^{+}(1+\eta)-\eta J_{z z}^{+}\right] .
\end{aligned}
$$

Was shown in the references $[5,11,25,26]$, if the zero modes or pair terms contribuitions are ignored, the rotational symmetry is breaking and the covariance are lost. After the inclusion of the zero modes, the full covariance of the current electromagnetic current is restored.

In the references $[19,20]$, are found some relations between the matrix elements of the plus component of electromagnetic current for the Z-terms contribuitions of the electromagnetic current:

$$
J_{x x}^{+Z}+\eta J_{z z}^{+Z}=0, \quad J_{z x}^{+Z}+\sqrt{\eta} J_{z z}^{+Z}=0 \quad \text { and } J_{y y}^{+Z}=0 .
$$




\section{EPJ Web of Conferences}

Table 1. The rho meson observables are calculated with the light-front quark model and compared with other model calculations. The units are, $\left(\mathrm{fm}^{2}\right)$ for the radius, $\left(e / 2 m_{V}\right)$ for the magnetic moment, $\left(e / m_{V}^{2}\right)$ for the quadrupole and $(\mathrm{MeV})$ the decay constant.

\begin{tabular}{|l|l|l|l|l|}
\hline \hline & $f_{\rho}$ & $\left\langle r_{\rho}^{2}\right\rangle$ & $\mu$ & $Q_{2}$ \\
\hline This work & 153 & 0.267 & 2.10 & 0.898 \\
{$[12]$} & & 0.310 & 2.69 & 0.840 \\
{$[17]$} & 221 & 0.540 & 2.01 & 0.410 \\
{$[21]$} & 129 & & 2.11 & \\
{$[27]$} & 134 & 0.296 & 2.10 & 0.910 \\
\hline \hline
\end{tabular}

The relation above, takes the following relations for the electromagnetic matrix elements for the valence components of the current whitout Z-terms: (the superscript $V$ indicates the valence terms):

$$
\begin{aligned}
J_{x x}^{+} & =J_{x x}^{+V}-\eta\left(J_{y y}^{+V}-J_{z z}^{+V}\right), \\
J_{z x}^{+} & =J_{z x}^{+V}-\sqrt{\eta}\left(J_{y y}^{+V}-J_{z z}^{+V}\right), \\
J_{z z}^{+} & =J_{y y}^{+V} \\
J_{z z}^{+Z} & =J_{y y}^{+V}-J_{z z}^{+V} .
\end{aligned}
$$

Using the above relationships in the Eq. (6), we obtain following expressions for the electromagnetic form factors of the spin-1 particles,

$$
\begin{aligned}
G_{0}^{B H} & =\frac{1}{3(1+2 \eta)}\left[J_{x x}^{+}(1+2 \eta)+J_{y y}^{+}(2 \eta-1)+J_{z z}^{+}(3+2 \eta)\right] \\
& =\frac{1}{3}\left[J_{x x}^{+V}+(2-\eta) J_{y y}^{+V}+\eta J_{z z}^{+V}\right], \\
G_{1}^{B H} & =\frac{1}{(1+2 \eta)}\left[\frac{J_{z x}^{+}}{\sqrt{\eta}}(1+2 \eta)-J_{y y}^{+}+J_{z z}^{+}\right] \\
& =\left[J_{y y}^{+V}-\frac{J_{z x}^{+V}}{\sqrt{\eta}}-J_{z z}^{+V}\right], \\
G_{2}^{B H} & =\frac{\sqrt{2}}{3(1+2 \eta)}\left[J_{x x}^{+}(1+2 \eta)-J_{y y}^{+}(1+\eta)-\eta J_{z z}^{+}\right] \\
& =\frac{\sqrt{2}}{3}\left[J_{x x}^{+V}-(1+\eta) J_{y y}^{+V}+\eta J_{z z}^{+V}\right],
\end{aligned}
$$

the final expressions for the electromagnetic form factors, $G_{0}^{C C K P}, G_{1}^{C C K P}$ and $G_{2}^{C C K P}$, given exactly the same expressions as Grach et al. [23] and the numerical calculation produce the same results compared with the instant form approach [20] (see the figures).

As shown before, in the references $[19,26]$ the elimination of the matrix element $I_{00}^{+}$, by the Inna Grach et al. [23], eliminates the zero modes, thus the result produced for the light-front approach calculations, same like the instant form formalism. In the table 1, the present work are compared with anothers approaches in the literature.

\section{Conclusion}

In the case of spin-1 particles, terms of no-valence becomes necessary to added [11, 20, 26, 27]. However, only term of the matrix elements of the electromagnetic current in the basis of the spin on the light-front $I_{00}^{+}$, contributes to the zero modes $[20,26]$. 

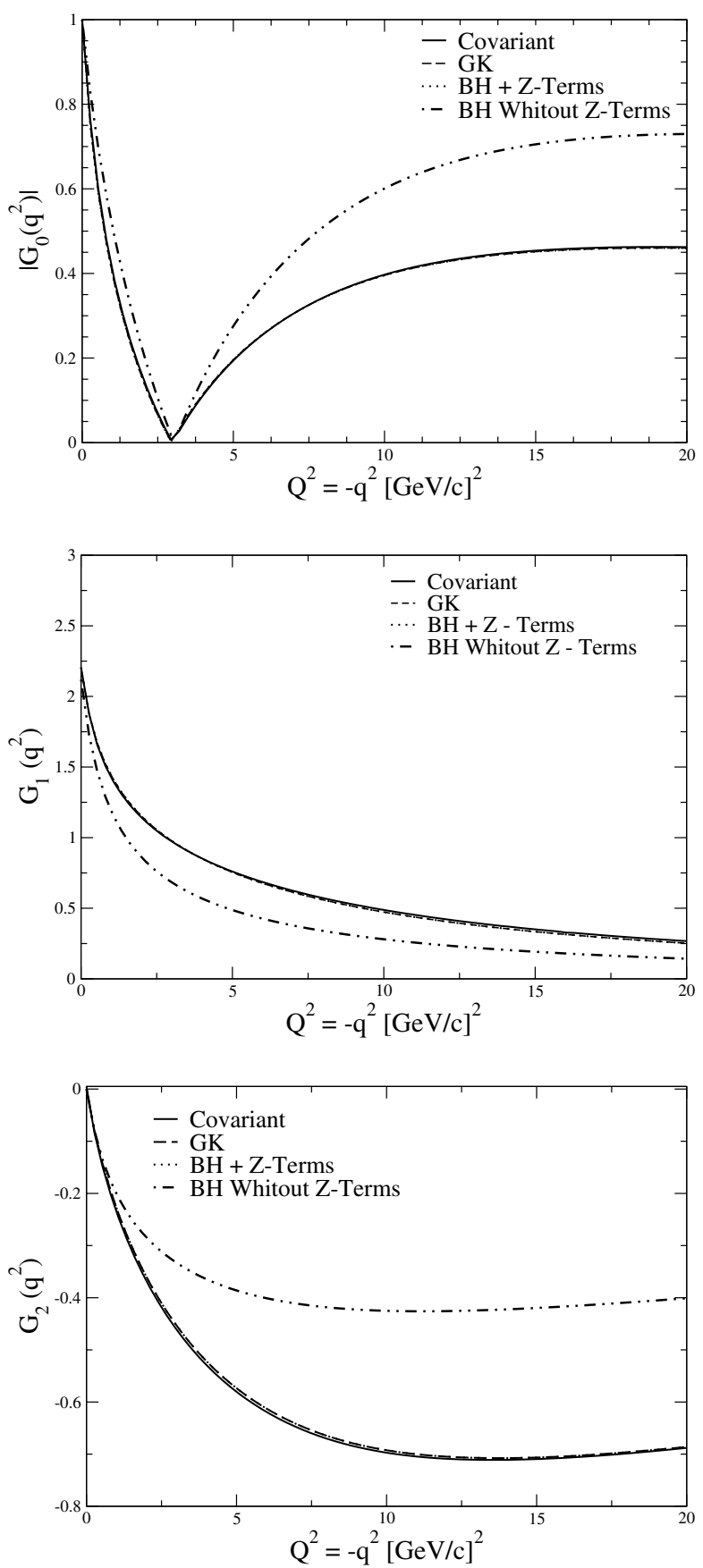

Figure 1. Electromagnetic charge form factor, $G_{0}$, for two prescriptions [23, 24] and compared with the instant form calculation. The prescriptions by the authors of the reference [24], after the inclusion of the zero modes contribuition, is the same of the covariant calculation and the prescriptions in the references [23].

Figure 2. Magnetic form factor, $G_{1}$, the labels are the same of the Fig. 1.

Figure 3. Quadrupole form factor, $G_{2}$, same labels like Fig. 1.

In this work, we use the relationships between some matrix elements of the electromagnetic current of the plus component, "+" for the rho meson [20], in order to get the electromagnetic form factors free of the zero modes.

We compared two existing prescriptions in the literature [23, 24] and the calculations are compared with instant form formalism. 
J. Pacheco B. C. de Melo thanks to the organizers MENU2013, Rome, Italy, for the invitation. This work was supported by the Brazilian agencies FAPESP (Fundação de Amparo a Pesquisa do Estado de São Paulo), CNPq (Conselho Nacional de Desenvolvimento Ciêntifico e Técnologico) and CAPES ( Coordenação de Aperfeiçoamento de Pessoal de Nível Superior).

\section{References}

[1] Taizo Muta, Foundations of Quantum Cromodynamics, An Introduction to Perturbative Methods in Gauge Theories (Word Scientific, Singapore, 1987)

[2] S. Brodsky, Hans-Cristian Pauli, Stephen Pinsky, Physics Reports 301, 299 (1998)

[3] P.A.M. Dirac, Rev. Mod. Physics 21, 392 (1949)

[4] P. Maris, C. Roberts, Phys. Rev. C 56, 3369 (1997)

[5] J.P.B.C. de Melo, H.W.L. Naus and T. Frederico, Phys. Rev. C 59, 2278 (1999)

[6] J.P.B.C. de Melo, T. Frederico, E. Pace and G. Salmè, Nucl. Phys. A 707, 399 (2002); ibid. Braz. J. Phys. 33, 301 (2003)

[7] Fabiano P. Pereira, J.P.B.C. de Melo, T. Frederico, Lauro Tomio, Nucl.Phys. A 610, 610 (2007)

[8] J.P.B.C. de Melo, T. Frederico, E. Pace and G. Salmè, Phys. Lett. B 581, 75 (2004); ibid. Phys. Rev. D 73, 074013 (2006)

[9] O. Leitner, J.-F. Mathiot, N. A. Tsirova, Eur. Phys. J. A 47, 17 (2011)

[10] Edson O. da Silva, J.P.B.C. de Melo, Bruno El-Bennich, Victo S. Filho, Phy. Rev. C 86, 038202 (2012)

[11] J.P.B.C. de Melo and T. Frederico, Phy. Rev. C 55, 2043 (1997)

[12] F.T. Hawes and M.A. Pichowsky, Phy. Rev. C 55, 2638 (1999)

[13] C. Adamuscin, G.I. Gakh and E. Tomasi-Gustafsson, Phys. Rev. C 75, 065201 (2007)

[14] Z.G. Wang and S.L. Wan, Phy. Rev. C 76, 025207 (2007)

[15] V.V. Braguta and A.I. Onishchenko, Phy. Rev. D 70, 033001 (2004)

[16] T. Frederico, E. Pace, Silva Pisano, G. Salmé, Nucl. Phys, B 199, 270 (2010)

[17] M.S. Bhagwat and P. Maris, Phy. Rev. C 77, 025203 (2008)

[18] H.M. Choi and C.R. Ji, Phys. Lett. B 696 (2011) 518

[19] J.P.B.C. de Melo and T. Frederico, Brazilian Journal of Physics, Vol. 34, no. 2A, 881 (2004)

[20] J.P.B.C. de Melo and T. Frederico, Phy. Lett. B 708, 87 (2012)

[21] M. Pitschmann, C.-Y. Seng, M.J.R.-Musolf, C.D. Roberts, S.M. Schmidt and D.J. Wilson, Phys. Rev. C 87, 015205 (2013)

[22] F. Cardarelli, I.L. Grach, I.M. Narodetskii, E. Pace, G. Salmé, S. Simula, Phy. Lett. B 349, 393 (1995)

[23] I.L. Grach and L.A. Kondratyuk, Sov. J. Nucl. Phys. 38, 198 (1984), ibid., I.L. Grach, L.A. Kondratyuk, and M.Strikman, Phys. Rev. Lett. 62, 387 (1989)

[24] S.J. Brodsky and J. Hiller, Phy. Rev. D 46, 2141 (1992)

[25] B.L.G. Bakker and C.R. Ji, Phy. Rev. D 65, 116001 (2002)

[26] Ho-Meoyng Choi and C.R. Ji, Phy. Rev. D 70, 053015 (2004)

[27] B.L.G. Bakker, H.M. Choi and C.R. Ji, Phy. Rev. D 65, 116001 (2002) 